\title{
Iron Chelation of Thalassemics in the Eastern Region of Morocco
}

\author{
A. Hassaine", A. Ghanam, A. Elouali, A. Babakhouya, M. Rkain, N. Benajiba
}

Pediatric Service-University Hospital Center Mohammed VI Oujda-Morocco

DOI: $10.36348 /$ sjmps.2020.v06i07.005

| Received: 04.07.2020 | Accepted: 11.07.2020 | Published: 24.07.2020

*Corresponding author: Amal Hassaine

\section{Abstract}

Thalassemia is a genetic disease of autosomal recessive inheritance, characterized by the reduction or absence of production of one of the normal globin chains, which causes chronic hemolytic anemia. Iron overload is the main prognostic factor for mortality and morbidity, and the quality of chelation remains the major prognostic factor for the disease. The aim of our work is to emphasize the major impact of chelating treatment in post-transfusion hemochromatosis, on life expectancy, the prevention of cardiac, hepatic and endocrine complications. Our study is a cross-sectional study on 15 cases of major $\beta$ thalassemia in children in the pediatric hematology unit of the CHU Oujda whose current age is between 2 and 13 years with an average of 8 years old and predominantly female (66\%). Therapeutically, all our patients are on a transfusion program every 2 to 4 weeks. The iron overload assessment in our series was based on the 3-month ferritinemia assay, liver MRI, and annual echocardiography. In our series, 14 of our patients were put on iron chelating treatment for which Deferasirox was the molecule of choice. All of our patients tolerated Deferasirox very well and did not tolerate Deferriprone. The evolution was marked by the improvement of ferritin in 7 cases, 4kept stationary ferritinemia and 3 cases increased their ferritinemia level. In the light of these results, thalassemia is a real public health problem whose complications are serious and whose morbidity and mortality are high, hence the need for good management for a good prognosis based on the 2 pillars monthly transfusion and iron chelation. Hematopoietic stem cell transplantation remains the only curative treatment.

Keywords: thalassemia major- Blood transfusion- Iron chelation- Ferritinaemea- Post transfusion hemochromatosis.

Copyright @ 2020: This is an open-access article distributed under the terms of the Creative Commons Attribution license which permits unrestricted use, distribution, and reproduction in any medium for non-commercial use (NonCommercial, or CC-BY-NC) provided the original author and source are credited.

\section{INTRODUCTION}

Thalassemia is an autosomal recessive genetic disease. It is characterized by the decrease or absence of production of one of the normal globin chains, which causes chronic hemolytic anemia. With sickle cell anemia, it represents the most frequent hereditary anemias in the world [1]. There are 3 types of B thalassemia: B thalassemia major requiring monthly transfusions, B thalassemia intermediate requiring little or no transfusion and B thalassemia minor not requiring transfusion. Besides the hematopoietic stem cell transplant, which is the only curative treatment of the major form, its management requires regular monitoring with an adequate transfusion program associated with iron chelation. The constant and early post-transfusion iron overload is the main prognostic factor for mortality and morbidity during TM. The management of this overload has been notably improved by the parallel development of orally active iron chelators and nuclear magnetic resonance imaging (MRI) techniques allowing the evaluation and monitoring under treatment of hepatic tissue iron and especially myocardial [2]. The aim of our work is to emphasize the major impact of chelating treatment in post-transfusion hemochromatosis, on life expectancy, the prevention of cardiac, hepatic and endocrine complications.

\section{Patients And Methods}

Our study is a cross-sectional study, covering 15 cases of major thalassemia $\mathrm{B}$, managed within the pediatric hematology unit of the Mohammed VI teaching hospital in Oujda. The protocol for therapeutic management was based essentially on monthly transfusions and iron chelation. The various complications linked to the disease and the undesirable effects of the therapies used were identified. The data study was carried out by SPSS software.

\section{Results}

Our study is a cross-sectional study of 15 cases of major $\beta$ thalassemia in children in the pediatric hematology unit of the Oujda University Hospital. The age of our patients at the time of diagnosis varied 
between 4 months and 5 years, with an average of 1.8 years. The current age of our patients is between 2 and 13 years with an average of 8 years. In our series, we noted a clear predominance of women ( $66 \%$ of cases). Most of our patients are originally far from the CHU where they have the capacity to take chelating treatment [Table I].

Therapeutically, all our patients are on a transfusion program every 2 to 4 weeks with an average interval between transfusions varying between 2 and 4 weeks with an average of 4 weeks. The evaluation of iron overload in our series is based on the dosage of ferritinemia every 3 months, the hepatic MRI $\left(\mathrm{T}_{2} *\right)$ performed rarely, the cardiac MRI not available from us and the annual echocardiography at the looking for a heart attack. Echocardiography demonstrated an impairment of diastolic function in only one case. In our series, 14 of our patients were put on iron chelating treatment for which Deferasirox was the molecule of choice. 13 cases were put on Deferasirox, 1 case on Deferiprone, One case has not yet been put on treatment since it is at the beginning of the diagnosis and 2 cases had been put on dual therapy one of which was on Deferasirox + Deferoxamine and the other was on Deferasirox + Deferiprone [Table II]. All of our patients tolerated Deferasirox very well without any side effects, while patients who were started on Deferriprone following the discontinuation of
Deferasirox reported arthralgia. A patient initially followed in another city far from the CHU and who was put on Défériprone presented an agranulocytosis with serious subcutaneous abscesses for which he was referred in our training (CHU) for additional management; the decision was to stop Deferiprone and the relay was made by Deferasirox, which was very well tolerated thereafter.

The evolution of the iron overload of our patients on chelating therapy was marked by the improvement of ferritinemia in 7 cases [figure 1], 4 patients kept stationary ferritinemia, one of which has been on dual therapy for one year (Désféral + Exjade) [Figure 2] and 3 patients increased their ferritinemias, one of which is on dual therapy (Exjade + Ferriprox) [Figure 3]. One patient has not yet been placed on a chelator due to a ferritinemia level of less than 1000ug / 1.

Patients who have improved their ferritinemia levels are very well looked after since they come to the hospital regularly and are very well taken care of [Figure 1]. 3 patients increased their ferritin levels, they are poorly managed and they do not take the chelating treatment since they live very far from the hospital and they do not come regularly due to lack of means and significant difficulties in transport material. These patients were lost to follow-up for a long time.

Table-I: Geographical origin of patients

\begin{tabular}{|c|c|c|}
\hline origin & Percentage & Distance between home /University Hospital Center \\
\hline Oujda & $26,6 \%$ & - \\
\hline Nador & $46,6 \%$ & $136,7 \mathrm{Km}$ \\
\hline Houcima & $13,3 \%$ & $260 \mathrm{Km}$ \\
\hline Guercif & $13,3 \%$ & $170 \mathrm{Km}$ \\
\hline
\end{tabular}

Table-2: Distribution of patients according to the iron chelator used.

\begin{tabular}{|c|c|}
\hline Chélateur du fer & Nombre de cas \\
\hline Deferasirox (Exjade) & 13 cases \\
\hline Deferiprone(Ferriprox) & 1 case \\
\hline Dual therapie & 2 cases \\
\hline Without traitement & 1 case \\
\hline
\end{tabular}

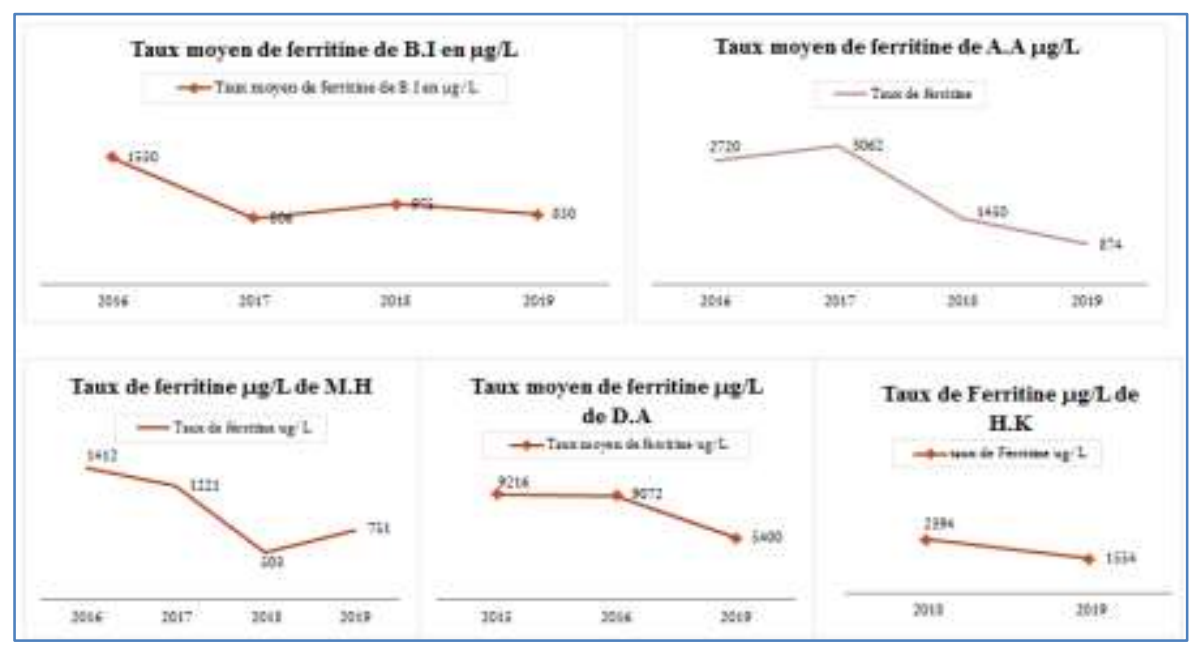

Fig-1: Distribution of patients who have reduced their ferritin levels 


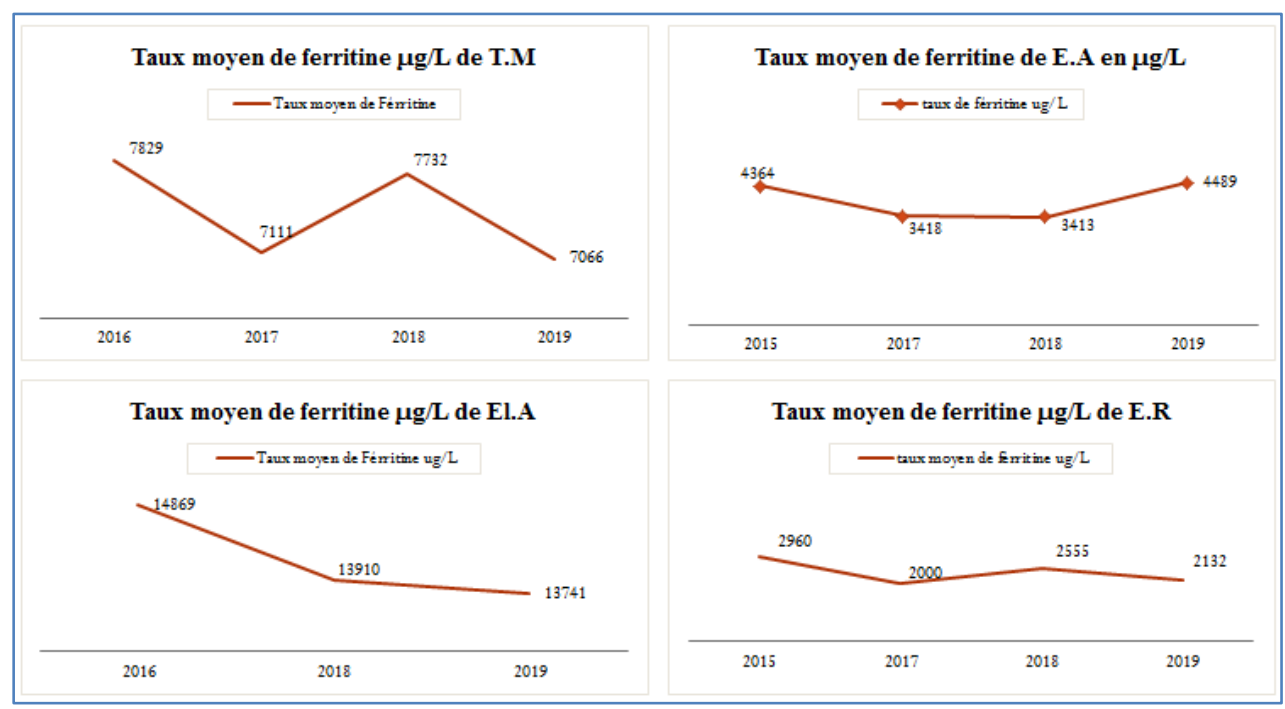

Fig-2: Distribution of patients who kept a stationary ferritinemia

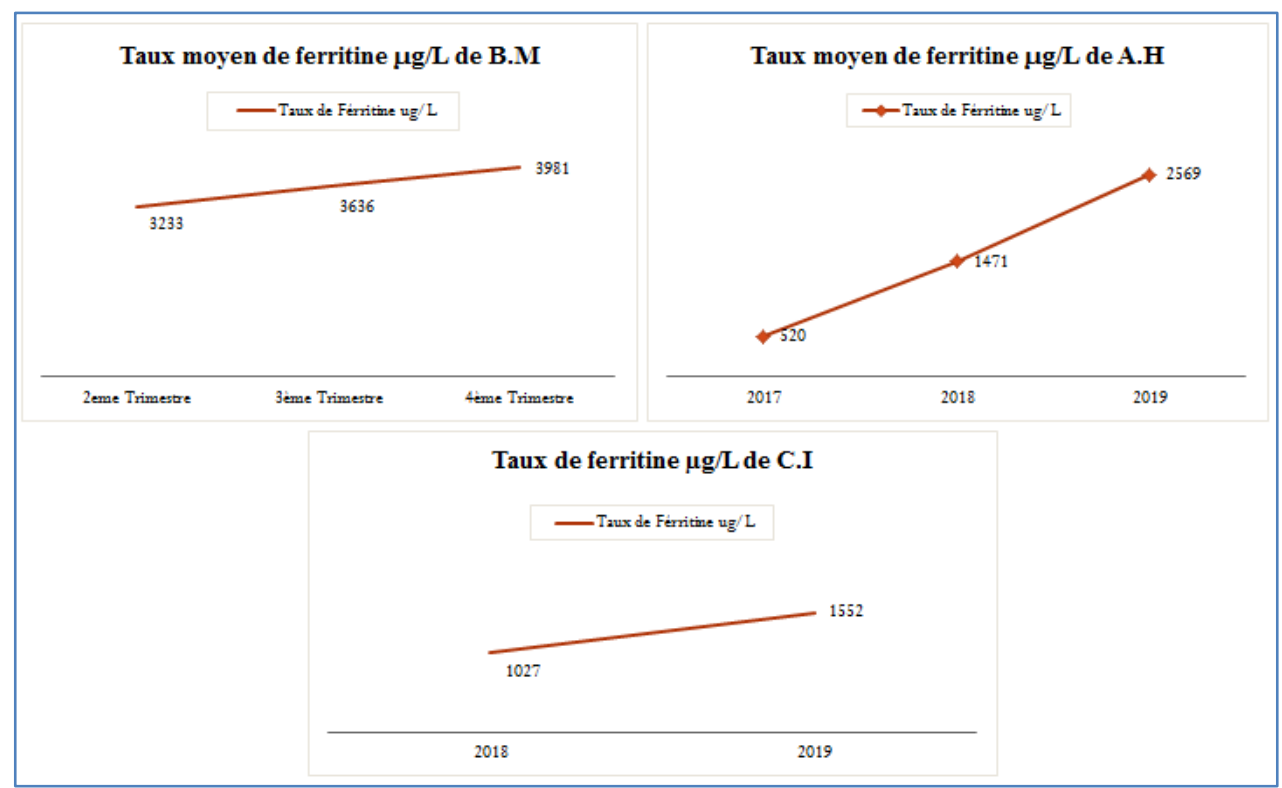

Fig-3: Distribution of patients who increased their ferritin levels

\section{DiscuSSION}

The aim of $\beta$-thalassemia treatment is to decrease anemia, and therefore to decrease the hyperstimulation of erythropoiesis and the secondary bone marrow expansion. It has two main components: conventional treatment and bone marrow transplantation [2]. Conventional treatment is based on three components: blood transfusion, iron chelation and splenectomy [2]. The most traditional transfusion regimen is the transition from a pre-transfusion $\mathrm{Hb}$ level from 9 to $10 \mathrm{~g} / \mathrm{dL}$ to a targeted post-transfusion rate at 14-15 g / dL. Blood transfusion is indicated for ferritinemia levels $>1000 \mathrm{Ug} / \mathrm{l}$, The interval between each transfusion is 2 to 4 weeks depending on the case [1]. In recent years, the management of posttransfusion iron overload has been notably improved by the parallel development of orally active iron chelators and nuclear magnetic resonance imaging (MRI) techniques allowing the evaluation and monitoring under treatment of hepatic and especially myocardial tissue iron [3]. Martial overload is all the more symptomatic since the number of concentrates administered is large and the course is long [4]. Iron chelation therapy involves the use of agents that bind to iron, make it chemically inert, and trigger a mechanism to excrete it. The purpose of this chelation is to prevent cardiac, hepatic and endocrine complications [5]. Treatment with chelators is started after 10 to 20 transfusions received, when the ferritin level reaches $1000 \mu \mathrm{g} / \mathrm{L}$. Iron chelators improve life expectancy.

\section{Several molecules are used} Deferoxamine (DFO):

Synthetic DFO (Désféral $\left.{ }^{\circledR}\right)$ is the first chelator used in $\beta$-thalassemia, used at a dosage of 40 to $50 \mathrm{mg} /$ $\mathrm{kg} / \mathrm{d}$. It is the standard chelating treatment used for more than 45 years. The treatment modality: night or 
daytime subcutaneous injection, continuous at 8 a.m. to 12 p.m., $40 \mathrm{mg} / \mathrm{kg} / \mathrm{d}$ for 5 to 7 days / week, via a pump or a portable infusor [6].

The general tolerance of the treatment is good, subject to early detection of its possible complications:

- Neurosensory disorders (optic neuritis and hearing loss) receiving large doses.

- Administration by high doses of the vein may cause respiratory distress.

- Locales Local reactions at the subcutaneous injection sites.

- Mycoses caused by certain fungi in the mucoraceae family.

In our series, only 1 patient was put on DFO, subcutaneously, very well tolerated. In the Fez series, 2 patients were put on Desferal (14.3\%). In the Ibn Sina Rabat Hospital series, $27 \%$ of the patients monitored do not take DFO regularly since the treatment is chronic and heavy. In France, 20 to $40 \%$ of patients are not compliant with DFO for the same reason.

\section{Deferiprone (DFP) (Ferriprox®)}

It is administered 3 times a day, at a dosage of 75 to $100 \mathrm{mg} / \mathrm{kg} /$ day [7]. The classic and mild effects are nausea, vomiting, abdominal pain, as well as brown discoloration of the urine with the risk of agranulocytosis. These serious side effects require immediate and permanent cessation of treatment, requiring substitution [1]. NFS should be performed once a week to detect the presence of agranulocytosis; reintroduction is contraindicated. A positive impact on the prevention of the occurrence of cardiac events and on cardiac mortality has also been reported in a very large retrospective study of thalassemic patients [6].

\section{Deferasirox (DFX) (Exjade®)}

This is the first line treatment. The effective dose is 20 to 30 or even $40 \mathrm{mg} / \mathrm{kg} /$ day. The least serious side effects are gastrointestinal upset. Patients also sometimes have skin rashes that disappear when treatment is stopped. Hypersensitivity reactions have been reported, as well as hematological conditions. The efficacy data for DFX treatment on cardiac overload are preliminary. In patients whose serum ferritin level has reached the target (between 500 and $1000 \mu \mathrm{g} /$ 1), dose reductions of 5 to $10 \mathrm{mg} / \mathrm{kg}$ should be considered in order to keep the serum ferritin level within the target range [2]. If the serum ferritin falls below $500 \mu \mathrm{g} / 1$, treatment should be discontinued.

\section{Dual therapies}

Combination therapy offers an alternative for patients whose iron overload is not sufficiently controlled with monotherapy. Dual therapies also reduce the frequency of DFO infusions by compensating with oral medication, which improves the quality of life for patients.

\section{Intensive chelation may be indicated before}

- A patient with severe cardiac hemochromatosis and / or cardiac dysfunction.

- Ferritinemia> $2500 \mu \mathrm{g} / 1$.

- Endocrine complications.

The molecules that can be used in intensive chelation are:

An oral chelator: Either as monotherapy:

Deferasirox (Exjade $\left.{ }^{\circ}\right): 30$ to $40 \mathrm{mg} / \mathrm{kg} /$ day in one dose.

Deferiprone (Ferriprox $\left.{ }^{\circledR}\right): 100 \mathrm{mg} / \mathrm{kg} / \mathrm{day}$ in 3 divided doses.

Or better dual therapy: Deferoxamine + Deferiprone // Deferasirox + Deferiprone.

An injectable chelator: DFO in continuous intravenous infusion: 50 to $100 \mathrm{mg} / \mathrm{kg} /$ day [2].

\section{CONCLUSION}

Thalassemia is the most common hereditary hemolytic anemia in the world. It is a real public health problem whose complications are serious and whose morbidity and mortality are high linked to these complications, hence the need for good care for a good prognosis based on the 2 pillars monthly transfusion and iron chelation. Hematopoietic stem cell transplantation remains the only curative treatment.

\section{Declaration of interest links}

The authors declare that they have no conflicts of interest.

\section{REFERENCES}

1. Epidemio-clinical, biological, therapeutic and progressive profile of thalassemia in childrenExperience of the pediatric hematology-oncology unit of the Hassan II teaching hospital in Fez (about 40 cases, 2016, thesis N 237/16.

2. PEC of iron overload during beta thalassemia. (2014). Experience of the hematology oncology unit of the Hassan II teaching hospital in Fez.

3. Hessissen, L., Harif, M. (2010). What new developments in thalassemia. Annals of Medicine and Therapeutics, 2(1): 14-24.

4. Perez, V. (2003). Beta thalassemia: General and etiology. Dissertation of Biochemistry PCEM 2.

5. Brittenham, G. M. (2011). Iron-chelating therapy for transfusional iron overload. New England Journal of Medicine, 364(2), 146-156.

6. Borgna-Pignatti, C., Cappellini, M. D., De Stefano, P., Del Vecchio, G. C., Forni, G. L., Gamberini, M. R., \& Cnaan, A. (2006). Cardiac morbidity and mortality in deferoxamine-or deferiprone-treated patients with thalassemia major. Blood, 107(9), 3733-3737.

7. Eliezer, A. (2016). Rachmilewitz; Patricia J. Giardina2 How I treat thalassemia by guest. 